\title{
ASSOCIATIONS OF ENZYMIC AND CHROMOSOMAL POLYMORPHISMS IN THE SEAWEED FLY, COELOPA FRIGIDA
}

\author{
T. H. DAY, T. DOBSON, P. C. HILLIER, D. T. PARKIN and BRYAN CLARKE \\ Department of Genetics, University of Nottingham, University Park, \\ Nottingham, NG7 2RD
}

Received 15.vi.81

\begin{abstract}
SUMMARY
Populations of the seaweed fly Coelopa frigida are polymorphic at three loci determining the enzymes peptidase-1 (Pep-1), alcohol dehydrogenase (Adh) and larval esterase-2 (Es-2). Alleles at these loci have been shown by others to be non-randomly associated with each other. In the present paper we report non-random associations between the $A d h$ and $E s-2$ loci and inversions on chromosome I. The two common alleles $A d h-B$ and $D$ are in strong linkage disequilibrium with the $\alpha$ and $\beta$ inversions, but the $A d h-A$ and $C$ alleles are not so. The $X$ and $Y$ alleles at the $E s-2$ locus show weak, but still significant, associations with the inversions. We consider possible linkage relationships of the loci on the chromosomal arrangements, and discuss the hypothesis that they constitute part of a coadapted gene complex whose members code for functionally related enzymes.
\end{abstract}

\section{INTRODUCTION}

POLYMORPHISMS for chromosomal inversions are found in many species of Diptera (see for example Brncic, 1970; Carson et al., 1970). One of the most obvious consequences of an inversion is the suppression or reduction of crossing-over in heterokaryotypes. This rather special genetical property has led to several suggestions about how inversion polymorphisms are maintained in populations. One possibility is that there is heterozygous advantage at one, several, or most of the loci contained within the inversion. A prolonged advantage of multiple heterozygotes could, in some circumstances, lead to the evolution of coadapted gene complexes (Dobzhansky, 1970). A second possibility is that the chromosomal arrangements (standard and inverted) accumulate deleterious recessive mutations because complementation, and the absence of recombination, prevent their selective elimination from the gene pool (Muller, 1964; Charlesworth, 1978).

A third, non-selective, interpretation of inversion polymorphisms follows from the unusual origin and properties of inversions. The first occurrence of an inversion is a unique event involving a particular collection of alleles that can thereafter only recombine at very low rates. "In other words, there is both an extremely small founder population and little potential for randomization of alleles within the inverted segment except through mutation or double recombination, both rare events" (Hedrick $e t$ al., 1978). Given these properties, an inversion polymorphism and its associated linkage disequilibrium may remain in a population for many generations. 
There have been several reports of enzyme-determining loci in linkage disequilibrium with chromosomal inversions (see reviews by Ishii and Charlesworth, 1977, and Hedrick et al., 1978). In attempting to explain these associations it is, in practice, extremely difficult to distinguish between the alternative explanations listed above, particularly when we have no detailed knowledge of the history of the population or species (in terms of population sizes, migration rates, etc.). However, a consistent and stable linkage disequilibrium between loci within an inversion is most economically interpreted as indicating the presence of coadapted gene complexes (Lewontin, 1974). The case is stronger if the loci concerned can be shown to interact epistatically. The gene products may, for example, operate together at a biochemical or physiological level so that selection acts on integrated groups of genes. A search for selection of this kind has been made by Mitton et al. (1973) using Mytilus edulis and by Zouros and Johnson (1976) using Drosophila mojavensis. We report here another possible example, the non-random association between two enzyme loci and a chromosomal inversion system in the seaweed fly Coelopa frigida.

C. frigida lives and breeds in piles of rotting seaweed ("wrack beds"), cast up on the seashore by the action of tides and storms. The general biology of the insect has been described by Dobson (1974) and Surver (1974). Electrophoretically detectable enzyme polymorphisms have been found in $C$. frigida at the alcohol dehydrogenase locus (Adh) (Kelsey, 1969; Day and Buckley, 1980), at a locus determining an adult peptidase (Collins, 1978), and at a locus determining a larval esterase (Es-2) (Hillier, Parkin and Clarke, in preparation). Collins (1978) has reported a nonrandom association between alleles at the $A d h$ and $P e p-1$ loci, and we have found that the $A d h$ and $E s-2$ loci are in linkage disequilibrium (Parkin, Hillier, Dobson and Clarke, in preparation).

British populations of $C$. frigida are polymorphic for several chromosomal inversions (Philip, 1958; Aziz, 1975). One of these, an overlapping triple inversion in chromosome $I$, is the subject of the present study. Intermediates between the standard $(\alpha)$ and the triple inverted $(\beta)$ sequences have not been found, and populations contain only three karyotypes $(\alpha \alpha, \alpha \beta$, and $\beta \beta)$. We have set out to test the possibility that the $A d h$ and $E s-2$ loci (and, by implication, $P e p-1$ as well) are associated with the $\alpha / \beta$ inversion system on chromosome I.

\section{MATERIALS AND METHODS}

(i) The animals

Larvae were collected from six natural populations inhabiting coastal sites in Britain. Our technique was to take 15-20 small handfuls of weed from places in different parts of the wrack bed, covering the whole area, and then to allow the larvae to intermix before taking a random sample for further study.

The sampling sites were as follows: Lyme Regis in Dorset (Ordnance Survey map reference SY338916), the Isle of Wight in Hampshire (O.S. SZ654872), Morfa Nefyn in Gwynedd (O.S. SH296408), Runswick Bay in North Yorkshire (O.S. NZ812158), Whitburn in Tyne and Wear (O.S. NZ408614) and Craster in Northumberland (O.S. NZ258201). The state 
of decomposition of the wrack beds varied widely, with the consequence that the populations contained grossly different proportions of animals at each of the developmental stages. The wrack beds at Lyme Regis, Whitburn and Craster were well decayed, and consequently relatively large fractions of the populations had pupated and eclosed when the samples of larvae were collected. Thus we cannot legitimately compare the frequencies of genotypes and inversions between different samples, because the inversions on chromosome I are known to have strong effects on development time (Day et al., 1980). The samples were collected only for the purpose of testing associations between enzyme loci and inversions. Legitimate comparisons between populations have already been reported by Kelsey (1969) and Collins (1978) and in the accompanying paper (Butlin et al., 1982).

\section{(ii) Chromosome preparation}

Salivary glands were removed from large third instar larvae, and the chromosomes were stained using the orcein squash technique (Darlington and La Cour, 1976), substituting propionic for acetic acid in the fixative and stain. The remainder of the animal was used for identifying allozymes (see below). At the beginning of our work, the first chromosomes were scored merely as homokaryotypes or heterokaryotypes. With more experience the two homokaryotypes $(\alpha \alpha$ and $\beta \beta$ ) could reliably be distinguished. Detailed banding maps of chromosome I are given by Aziz (1975).

\section{(iii) Electrophoresis of allozymes}

Electrophoresis in starch gels was used to identify the genotypes of animals at the alcohol dehydrogenase (Adh) and esterase-2 (Es-2) loci. The buffer systems and staining methods for $\mathrm{ADH}$ are described by Day et al. (1974). Five alleles have been identified at this locus, and the $A d h$ $B, C$ and $D$ alleles normally occur in natural populations at frequencies greater than 0.05. Using the method of Gahne (1966), four Es-2 allozymes were resolved. The Es-2 $X$ and $Y$ alleles are common but $E s-2 V$ and $W$ are very much rarer, at frequencies usually less than $0 \cdot 05$.

\section{RESUlts}

\section{(i) Association of Adh and Es-2 alleles}

Following the classification of individual animals for both their alcohol dehydrogenase (ADH) and their esterase (Es-2) allozymes it has become clear that there is a strong linkage disequilibrium between the two loci. Data from several populations will be described in detail elsewhere (Parkin, Hillier, Dobson and Clarke, in preparation), but a typical sample, from Runswick Bay, is summarized in table 1 . The rare $E s-2 V$ and $W$ alleles and the very rare $A d h-A$ allele have been omitted from the table. The very significant departure from randomness can largely be attributed to excesses of $A d h-B$ with $E s-2 Y$ and of $A d h-D$ with $E s-2 X$. There is little obvious association between the $A d h-C$ allele and any allele at the Es-2 locus. 
TABLE 1

The Adh and Es-2 genotypes of 524 larvae collected at Runswick Bay

\begin{tabular}{lccccccc} 
& & \multicolumn{7}{c}{ Adh genotypes } \\
Esterase-2 & & $B B$ & $B C$ & $B D$ & $C C$ & $C D$ & $D D$ \\
genotypes & $X X$ & 8 & 13 & 59 & 6 & 46 & 74 \\
& & $* * *$ & $* *$ & $*$ & & $\dagger+\dagger$ & $\dagger+\dagger$ \\
& & 41 & 27 & 130 & 6 & 16 & 7 \\
& $Y Y$ & 49 & 17 & 13 & 0 & 1 & $* * *$ \\
& & $\dagger+\dagger$ & $\dagger$ & $* *$ & & $* *$ & $* *$
\end{tabular}

Animals possessing one or more of the rare $E s-2 v$ or $W$ alleles or the very rare $A d h-A$ allele have not been included. Stars indicate that there were significantly fewer animals observed than would be expected if there was random association of alleles at two loci. Daggers indicate that significantly more animals were observed than expected. One, two or three stars or daggers indicate that the differences are significant at the levels of 5 per cent, 1 per cent and $0 \cdot 1$ per cent respectively. A full description and analysis of these data will be reported elsewhere (Parkin, Hillier, Dobson and Clarke, in preparation).

\section{(ii) Association between Adh and chromosome I}

Four hundred and two larvae from six different populations were scored for their inversion karyotype and at least one enzyme genotype. The complete data are given in the appendix. Tables 2 and 3 show the combinations of $\boldsymbol{A d h}$ alleles and chromosomal types in all our samples. The data are grouped into those recorded before (table 2) and after (table 3 ) the two homokaryotypes could be distinguished. Both sets of data show clearly

\section{TABLE 2}

Combinations of Adh genotypes and chromosome I karyotypes recorded before the individual homokaryotypes could be distinguished. The animals came from the Runswick and Whitburn populations. The starred individuals are exceptions to the tight association between the B and $\mathrm{D}$ alleles and the $\alpha$ and $\beta$ chromosome forms

\begin{tabular}{lcccccccccc} 
Chromosome-I & \multicolumn{10}{c|}{ Adh genotype } \\
$\quad$ karyotype & $A A$ & $A B$ & $A C$ & $A D$ & $B B$ & $B C$ & $B D$ & $C C$ & $C D$ & $D D$ \\
Homokaryotypic & 0 & 0 & 0 & 2 & 74 & 9 & $3^{*}$ & 1 & 1 & 11 \\
Heterokaryotypic & 0 & 0 & 0 & 2 & $1^{*}$ & 5 & 108 & 1 & 4 & $3^{*}$
\end{tabular}

TABLE 3

Combinations of Adh genotypes and chromosome-I karyotypes recorded after the individual homokaryotypes could be distinguished. The animals came from the Lyme Regis, Isle of Wight, Craster and Morfa Nefyn populations

$\begin{array}{lcccccccccc}\begin{array}{l}\text { Chromosome-1 } \\ \text { karyotype }\end{array} & A A & A B & A C & A D & B B & B C & B D & C C & C D & D D \\ \begin{array}{c}\text { Homokaryotype } \\ \quad \alpha \boldsymbol{\alpha}\end{array} & 0 & 5 & 2 & 0 & 39 & 16 & 0 & 0 & 0 & 0 \\ \begin{array}{c}\text { Homokaryotype } \\ \boldsymbol{\beta} \boldsymbol{\beta}\end{array} & 0 & 0 & 0 & 0 & 0 & 0 & 0 & 0 & 1 & 8 \\ \begin{array}{c}\text { Homokaryotypic } \\ \text { but not identified }\end{array} & 0 & 0 & 0 & 0 & 9 & 8 & 0 & 0 & 0 & 2 \\ \begin{array}{c}\text { Heterokaryotype } \\ \boldsymbol{\alpha} \boldsymbol{\beta}\end{array} & 0 & 0 & 0 & 2 & 0 & 5 & 53 & 0 & 15 & 0\end{array}$


that there are non-random arrays of combinations. We must emphasise that the associations shown in tables 2,3 and 5 are not the consequence of grouping several populations together, as can be seen from the original data in the appendix. There are highly significant deviations from random associations within all the individual populations, and the deviations are consistent in direction. The groupings have been made for clarity of exposition rather than for the analysis. In table 3 there are absolute associations of the $A d h-B$ allele with the $\alpha$ inversion, and of the $D$ allele with the $\beta$ inversion. These associations are also obvious in the Runswick and Whitburn animals (table 2), but there are seven exceptional individuals (starred). It is impossible to say whether they are true exceptions or scoring errors. If they are true exceptions, then overall (summing the data in tables 2 and 3 ) seven out of $622(1.1$ per cent) of the $A d h-B$ and $D$ alleles are found in the "wrong" association. These exceptional animals were found only in the Runswick and Whitburn populations. They were not found in the nearby Craster population, nor in the other three populations.

We believe that these exceptions are scoring errors. It is worth noting that their distribution differs significantly between tables 2 and 3 (Fisher's exact test $\longrightarrow$ one tailed; $p=0 \cdot 045$ ).

$\begin{array}{ccc} & \begin{array}{c}\text { "Normal" } \\ \text { associations }\end{array} & \begin{array}{c}\text { "Exceptional" } \\ \text { associations }\end{array} \\ \text { Table 2 } & 393 & 7 \\ \text { Table 3 } & 222 & 0\end{array}$

Thus the absence of exceptional animals in table 3 does not appear to be a chance occurrence. It may be the result of improvement in the chromosomal preparations. The exceptions are not associated with any particular $E s-2$ genotype, and they are found in both the samples included in table 2 (see appendix).

The $A d h-C$ allele does not show a strong association with the inversions. It occurs with both types. If we assume that the $B$ and $D$ alleles are indeed uniquely associated each with its "own" inversion, it is possible to establish with which inversion the $C$ allele is associated in $B C$ and $C D$ heterozygotes, and so to test whether $C$ associates at random with $\alpha$ and $\beta$. For example, we can infer that $B C$ homokaryotypic animals must have the $C$ allele associated with the $\alpha$ inversion, and that $B C$ heterokaryotypic individuals must have $C$ associated with the $\beta$ inversion. The numbers of $C \alpha$ and $C \beta$ obtained in this way are given in table 4 .

TABLE 4

The numbers of $\mathrm{Adh}-\mathrm{C}$ alleles found in combination with each chromosomal type (see text)

$\begin{array}{lrr} & C_{\alpha} & C_{\beta} \\ \text { Lyme Regis } & 11 & 3 \\ \text { Isle of Wight } & 3 & 2 \\ \text { Morfa Nefyn } & 17 & 1 \\ \text { Runswick Bay } & 0 & 1 \\ \text { Whitburn } & 14 & 6 \\ \text { Craster } & 8 & 0 \\ \quad \text { Total } & 53 & 13\end{array}$


We have used Fisher's exact $2 \times 2$ test (two-tailed) to find whether there are significant differences in the frequencies of the two combinations between the six samples. Of the 15 pairwise comparisons none is statistically significant (in every case $p>0 \cdot 12$ ). It therefore appears that the samples are homogeneous. A conventional analysis by $\chi^{2}$ is possible according to the criterion of Lewontin and Felsenstein (1965) if the Runswick Bay sample is excluded. The resulting $\chi^{2}$ for heterogeneity $\left(\chi_{(4)}^{2}=7 \cdot 21 ; p>\right.$ 0.10 ) is not significant.

One way of enquiring if the $A d h-C$ allele associates at random with the two chromosomal forms would be to compare, in each sample, the $C \alpha: C \beta$ with the $\alpha: \beta$ ratio. However, the observed $\alpha: \beta$ ratio excludes unclassified homokaryotypes, which contain an excess of $\beta \beta$ genotypes (table 2), and so probably of the $\alpha$ inversion. We can avoid this problem by comparing the $C \alpha: C \beta$ ratio with the $A d h-B: A d h-D$ ratio. Since the latter ratio is significantly heterogeneous between localities, the comparisons must be made individually by locality. None is significant, nor is the combined comparison $(\Sigma \chi)^{2} / n=\chi_{(1)}^{2}=2 \cdot 18$. There is thus no evidence that the $C$ allele is associated with a particular chromosome.

The $A d h-A$ allele is also found in both inversions. If the numbers of $A \alpha$ and $A \beta$ are calculated in the same way as the numbers of $C \alpha$ and $C \beta$, we find a total ratio (over all localities) of $9 A \alpha: 2 A \beta$. The numbers are too small to carry out individual comparisons, and therefore provide no evidence that the $A$ allele is associated with a particular chromosome. To summarise, it seems that the $A d h-B$ allele is always associated with the $\alpha$ form of the chromosome, $A d h-D$ with the $\beta$ form, and $A d h-C$ and $A d h-A$ with either at random (although in the last two cases larger samples might detect departures from randomness).

\section{(iii) Association between Es-2 and chromosome I}

From table 5 it seems that the Es-2 locus is also associated with the $\alpha / \beta$ inversion system. At three localities we have scored both $\alpha \alpha$ and $\beta \beta$ homokaryotes, and in all three there is a suggestion that the Es-2 $Y$ allele is associated with the $\alpha$ chromosome, and the $E s-2 X$ allele with the $\beta$ chromosome. Only in the sample from Lyme Regis is the association significant $\left(\chi_{(1)}^{2}\right.$ with Yates' correction $\left.=5.60, p<0.02\right)$. At three localities (Morfa Nefyn, Runswick Bay, and Whitburn), however, there is a significant

TABLE 5

The association between Es-2 and chromosome I. Data from all six populations were combined. No animals with the genotypes VV, VW or WW were examined cytologically

Es-2 genotype

$\begin{array}{cccccccc}\begin{array}{c}\text { Chromosome I } \\ \text { karyotype }\end{array} & V X & V Y & W X & W Y & X X & X Y & Y Y \\ \begin{array}{c}\text { Homokaryotype } \\ \alpha \alpha\end{array} & 0 & 1 & 0 & 0 & 3 & 31 & 28 \\ \begin{array}{c}\alpha{ }^{\prime} \\ \begin{array}{c}\beta \beta \\ \text { Homokaryotype }\end{array}\end{array} & 2 & 0 & 1 & 0 & 6 & 0 & 0 \\ \begin{array}{c}\text { unclassified } \\ \text { Heterokaryotype } \\ \alpha \beta\end{array} & 5 & 5 & 4 & 2 & 23 & 34 & 46 \\ \alpha \beta & 8 & 10 & 5 & 1 & 43 & 119 & 15\end{array}$


relationship between heterozygosity for $E s-2 X / E s-2 Y$ and for $\alpha / \beta\left(\chi_{(1)}^{2}\right.$, with Yates' correction $7 \cdot 56,12.90$ and 7.00 respectively) and the other three localities show the same tendency. The $E s-2 V$ and $W$ alleles are too rare to test for such associations. However, heterozygotes possessing the $V$ allele occur with both $\alpha \alpha$ and $\beta \beta$ karyotypes. The association of the Es-2 locus with chromosome I is evidently real, but weaker than that of the Adh locus.

\section{Discussion}

The chromosomal cytology of Coelopa frigida has been described in detail by Aziz (1975). Chromosome I carries three overlapping inversions, chromosome II has a single inversion and chromosome III is polymorphic for three non-overlapping inversions. It seems likely that the inversion system on chromosome I will create three regions within which loci will exhibit different patterns of recombination (see fig. 1). Loci within the

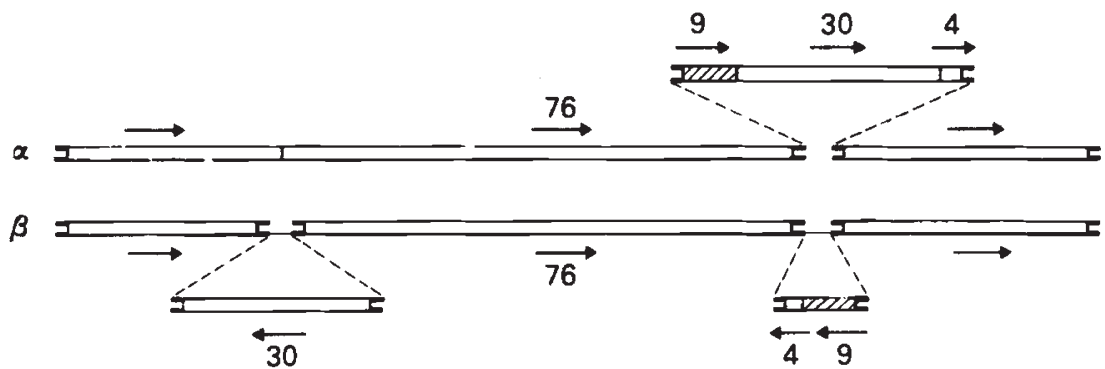

FIG. 1.-The chromosomal arrangements of the $\alpha$ and $\beta$ inversions on the right arm of chromosome I (redrawn from Azis, 1975). The figures give the numbers of bands (some of them doublets) in each chromosome segment, and the arrows show the relative polarities of the chromosomal segments.

relocated segments (segments with 4, 9 or 30 bands) might be expected to recombine very rarely indeed, if at all. Crossovers could occur only if the chromosome became twisted in a very complex way. Loci in the central paired region (with 76 bands), however, might occasionally be involved in crossovers. Single crossovers would generate meiotic products that were either duplicated or deficient for the 30 band segment. It seems likely that such recombinants would be lethal. Double crossovers, however, would not result in duplications or deficiencies, and would allow the recombination of alleles at loci between the two sites of breakage. Thus we might expect that genes located in the centre of the paired region would exhibit less extreme linkage disequilibrium than those in the relocated segments. The third region of interest is that containing loci lying outside the triple inversion, but still within the zone where crossing over is inhibited. Such loci would be expected to exhibit degrees of linkage disequilibrium correlated with their proximity to the inversion.

There are two possible methods of determining in which of these regions a specified locus occurs. Unequivocal maps could be made by using nucleic acid hybridization in situ. This would require pure messenger RNA 
transcribed from the relevant locus, in order to produce cloned cDNA. No such probe has yet been obtained from $C$. frigida, although cDNA from the Adh locus of Drosophila melanogaster may become available in the near future.

An alternative is to use classical recombination mapping. There is a difficulty, however, in obtaining strains homozygous for particular alleles at the loci to be studied. Our crosses have shown that there is little if any recombination between the $A d h$ and $E s-2$ loci in heterokaryotypes. (Among 760 progeny scored there were no recombinants.) The difficulty of synthesizing required genotypes in laboratory crosses may require us to find them in natural populations, but because the Es-2 genotypes can only be scored in larvae, and the Pep-1 genotypes in adults, it will be a laborious exercise. We are postponing the search until we have found two or three more loci associated with the inversion.

Despite these problems, the chromosome I inversion system offers a useful opportunity to study the causes of linkage disequilibrium. There are several pieces of evidence, none of them unequivocal, that encourage us to think in terms of a selective explanation for the disequilibrium. The first is a remarkable uniformity in the frequencies of the chromosomal inversions in all the British populations that have been studied (Collins, 1978; Butlin et al., 1982). The second is a profound, and almost inevitably selective, association of the $A d h$ locus with variations in development time (Day et al., 1980). The third is the existence of apparent heterozygous advantage, also associated with the Adh locus (Kelsey, 1969; Collins, 1978). The fourth is the observation that of the three loci known to be associated with the inversion system on chromosome I, two are probably concerned with extracellular digestion, and the other is possibly so. Peptidase-1 is a gut enzyme capable of acting on a wide range of dipeptides (Collins, 1978). Esterase-2 has a wide spectrum of substrate specificities for esters present in the gut lumen, and can also hydrolyse several short-chain fatty acids (Hillier and Day, unpublished data). ADH appears to be located in the haemocoele rather than the gut lumen, but it acts on alcohols, which are the products of esterase digestion. The fact that the enzymes in linkage disequilibrium with a chromosomal inversion also seem to be functionally related encourages us to look at other enzymes that may be similarly related. If we find, for example, that amylases, glycosidases or lipases are determined by loci in or near the inversion, then the evidence for a coadapted gene complex would become more convincing.

Acknowledgments.-We are very grateful to the Nuffield Foundation and the Science Research Council for financial support.

\section{REFERENCES}

AZIZ, J. B. 1975. Investigations into chromosomes 1, 2 and 3 of Coelopa frigida (Fab.). Ph.D. Thesis, University of Newcastle upon Tyne.

BRNCIC. D. 1970. Studies on the evolutionary biology of Chilean species of Drosophila. In Essays in Evolution and Genetics in Honour of Theodosius Dobzhansky, eds. M. K. Hecht and W. C. Steere, pp. 401-436. North Holland Publ. Co., Amsterdam.

BUTLIN, R. K., COLLINS, P. M., SKEVINGTON. S. J., AND DAY. T. H. 1982. Genetic variation at the alcohol dehydrogenase locus in natural populations of the seaweed fly, Coelopa frigida. Heredity, 48, 45-55. 
CARSON, H. L., HARDY, D. E., SPIETH, H. T., AND STONE, W. S. 1970. The evolutionary biology of the Hawaiian Drosophilidae. In Essays in Evolution and Genetics in Honour of Theodosius Dobzhansky, eds. M. K. Hecht and W. C. Steere, pp. 437-543. North Holland Publ. Co., Amsterdàm.

CHARLESWORTH, B. 1978. Model for evolution of Y chromosomes and dosage compensation. Proc. natn. Acad. Sci. USA, 75, 5618-5622.

COLlins, P. M. 1978. Studies on genetic polymorphism in Coelopa frigida. Ph.D. Thesis, University of Nottingham.

DARLINGTON, C. D., AND LA COUR, L. F. 1976. The Handling of Chromosomes, 6th edn, p. 124. Allen and Unwin Ltd., London.

DAY, T. H., AND BUCKLEY, P. A. 1980. The alcohol dehydrogenase polymorphism in the seaweed fly, Coelopa frigida. Biochem. Genet., 18, 727-742.

DAY, T, H., HILliER, P. C., AND CLARKE, B. 1974. Properties of genetically polymorphic isozymes of alcohol dehydrogenase in Drosophila melanogaster. Biochem. Genet., 11, 141-153.

DAY, T. H., DOBSON, T., HillieR, P. C., PARKIN, D. T., AND ClARKE, B. 1980. Different rates of development associated with the alcohol dehydrogenase locus in the seaweed fly, Coelopa frigida. Heredity, 44, 321-326.

DOBSON, T, 1974. Studies on the biology of the kelp-fly Coelopa in Great Britain. J. Nat. Hist., 8, 155-177.

Dobzhansky, T. 1970. Genetics of the Evolutionary Process. Columbia University Press, New York.

GAHNE, B. 1966. Studies on the inheritance of electrophoretic forms of transferrins, albumins, pre-albumins and plasma esterases of horses. Genetics, 53, 681-694.

HEDRICK, P., JAIN, S., AND HOLDEN, L. 1978. Multilocus systems in evolution. Evol. Biol., $11,101-184$.

ISHII, K., AND CHARLESWORTH, B. 1977. Associations between allozyme loci and gene arrangements due to hitch-hiking effects of new inversions. Genet. Res., 30, 93-106.

KELSEY, M. C. 1969. An investigation of the alcohol dehydrogenase polymorphism in Coelopa frigida (Fab.). B.Sc. Thesis, University of Edinburgh.

LEwontin, R. C. 1974. The Genetic Basis of Evolutionary Change. Columbia University Press, New York.

LEWONTIN, R. C, AND FELSENSTEIN, J. 1965. The robustness of homogeneity tests in $2 \times N$ tables. Biometrics, 21, 19-33.

MITTON, J. B., KOEHN, R. K., AND PROUT, T. 1973. Population genetics of marine pelecypods. III. Epistasis between functionally related isoenzymes of Mytilus edulis. Genetics, 73, 478-496.

MULLER, H. J. 1964. The relation of recombination to mutational advance. Mutation Res., 1, 2-9.

PHILIP, U. 1958. Genetics and cytology of the seaweed fly, Coelopa frigida (Fab.). Proc. Xth Int. Congr. Genet. Montreal, 2, p. 217.

SURVER, W. M. 1974. Observations on some aspects of the biology of the kelp-fly: Coelopa frigida (Fabricius). Ph.D. Thesis, University of Notre Dame, Indiana.

ZOUROS, E., AND JOHNSON, W. 1976. Linkage disequilibrium between functionally related enzyme loci of Drosophila mojavensis, Can. J. Genet. Cytol., 18, 245-254.

\section{APPENDIX}

The chromosome I karyotypes and Adh and Es-2 genotypes of animals from six populations. Rare combinations of genotypes from the Morfa Nefyn and Whitburn samples are listed separately

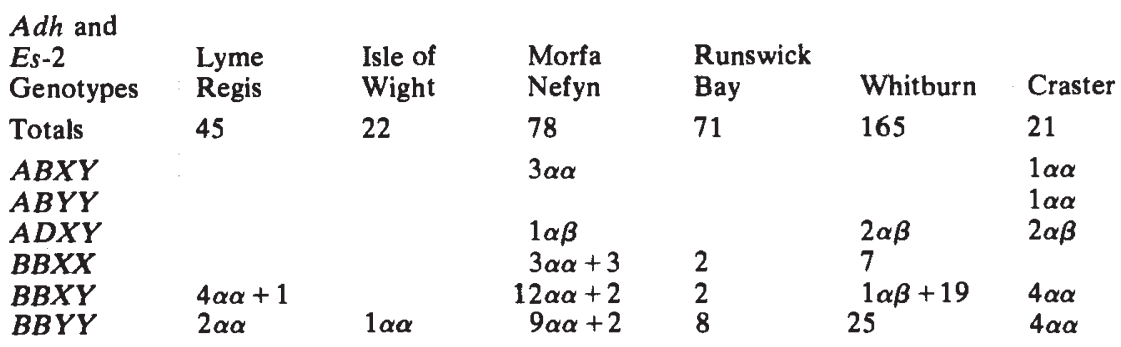




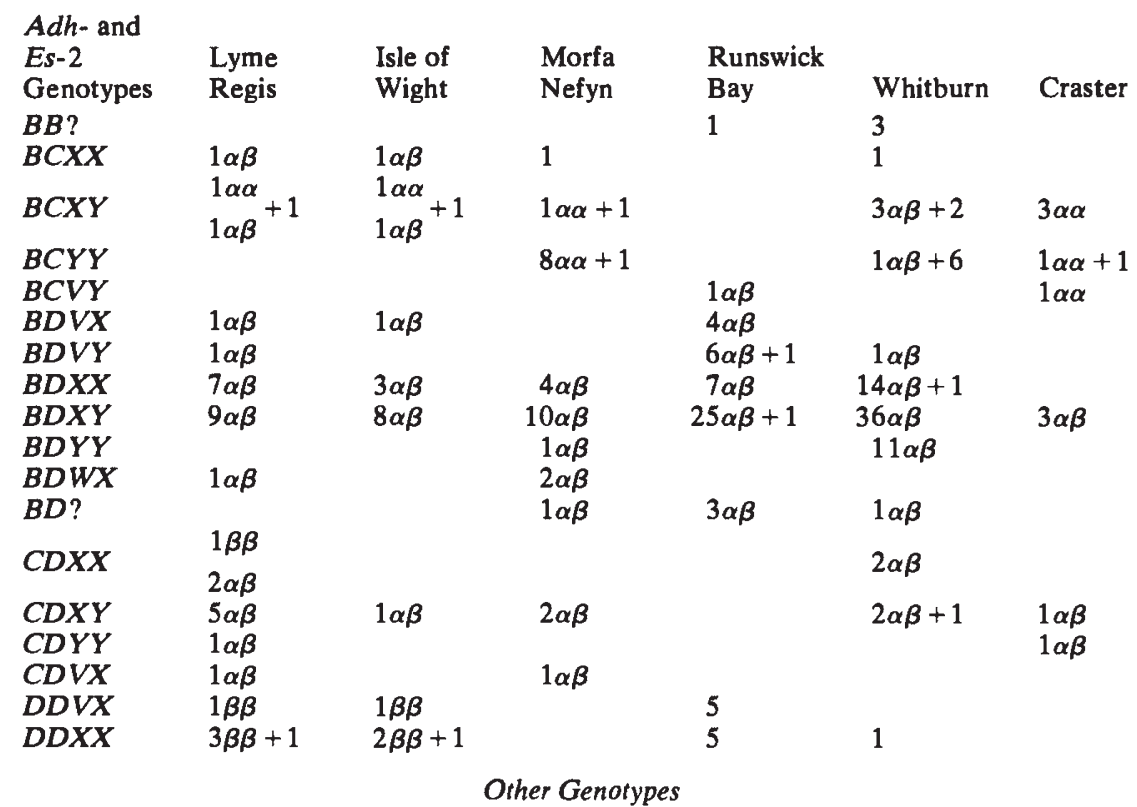

Morfa Nefyn

$\begin{array}{ll}1 A C X Y \alpha \alpha & 1 B C ? \text { hom } \\ 1 A C Y Y \alpha \alpha & 1 B D W Y \alpha \beta \\ 1 A D V Y \alpha \beta & 1 D D W X \beta \beta \\ 1 B C W X \alpha \beta & 1 ? Y Y \alpha \alpha \\ 1 B C W X \text { hom } & \end{array}$

Whitburn

$\begin{array}{lll}\text { 1ADXYhom } & 1 C C X Y \alpha \beta & 1 \text { ? WXhom } \\ 1 A D Y Y h o m & 1 C C Y Y h o m & 2 ? X X \alpha \beta \\ 3 B B \text { Whom } & 3 D D X Y \alpha \beta & 4 ? X Y \alpha \beta \\ 2 B B W Y h o m & 1 ? V Y h o m & 2 ? X Y h o m \\ 2 B B V Y h o m & 1 ? \text { WX } \alpha & \end{array}$

$\alpha \alpha=\alpha \alpha$ homokaryotypes

$\beta \beta=\beta \beta$ homokaryotypes

$\alpha \beta=\alpha \beta$ heterokaryotypes

$+=$ Unclassified homokaryotypes

$?=A d h$ or Es-2 genotype was unscorable 